\title{
Retrieving leaf area index from multi-angular airborne data
}

\author{
Francisco Javier García-Haro, Fernando Camacho-de Coca and Joaquín Meliá \\ Department of Thermodynamics, University of Valencia, Spain
}

\begin{abstract}
This work is aimed to demonstrate the feasibility of a methodology for retrieving bio-geophysical variables whilst at the same time fully accounting for additional information on directional anisotropy. A model-based approach has been developed to deconvolve the angular reflectance into single landcovers reflectances, attempting to solve the inconsistencies of 1D models and linear mixture approaches. The model combines the geometric optics of large scale canopy structure with principles of radiative transfer for volume scattering within individual crowns. The reliability of the model approach to retrieve LAI has been demonstrated using data from DAISEX99 campaign at Barrax, Spain. Airborne data include POLDER and HyMap data in which various field plots were observed under varying viewing/illumination angles. Nearly simultaneously, a comprehensive field data set was acquired on specific crop plots. The inversions provided accurate LAI values, revealing the model potential to combine spectral and directional information to increase the likely accuracy of the retrievals. In addition, the sensitivity of retrievals with the angular and spectral subset of observations was analysed, showing a high consistency between results. This study has contributed to assess the uncertainties with products derived from satellite data like SEVIRI/MSG.
\end{abstract}

Key words $L A I-$ inversion - POLDER - HyMap multi-angular

\section{Introduction}

The LSA SAF Project is part of the ground segment for the EUMETSAT missions METEOSAT Second Generation (MSG) and European Polar System (EPS), developed by the ESA. Our aim is to develop robust and operational algorithms for retrieving vegetation parameters from the synergistic use of SEVIRI/MSG and AVHRR-3/EPS. These instruments will offer innovative angular capabilities for determining vegetation products over Europe and Africa thanks to concomitant multiple viewing and illumination

Mailing address: Dr. Francisco Javier García-Haro, Department of Thermodynamics, University of Valencia, C/Dr. Moliner 50, 46100 Burjassot, Valencia, Spain; e-mail: J.Garcia.Haro@uv.es geometries (Van-Leeuwen and Roujean, 2002). Remotely sensed BRF data are the only way to monitor the vegetation on a global scale. Traditional approaches rely on the exploitation of the reflectance variations in the spatial, temporal and spectral domains, in lack of directional information. However, observations demonstrate that the anisotropic behaviour of the surface is an important property and the absence of angular information inevitably produces a bias in the parameter evaluation (Roujean et al., 1992; Leblanc et al., 1997). Our main concern is to retrieve fractional Leaf Area Index (LAI) corrected from the effects of the sun-target-sensor geometry.

Linear mixture is the basis hypothesis in some simple BRDF (Bidirectional Reflectance Distribution Function) models (e.g., geometricaloptical or kernel-driven models) for simulation of scene reflectance or in remote sensing algorithms, e.g., Spectral Mixture Analysis (SMA). However, this technique is ineffective to address the presence of multiple scattering, surface anisotropy and the scaling processes (Qin and 
Gerstl, 2000). Most BRDF one-dimensional models imply surface homogeneity within an image pixel and are, therefore, limited to address mixed landcovers pixels, which are common in coarse resolution satellites. Different hybrid models have been developed in recent years to describe the radiation regime in forest canopies $(\mathrm{Li}$ and Strahler, 1992; Chen and Leblanc, 1997). These models assume a medium consisting of gaps and regions idealized by a turbid volume with a foliage density of small leaves. In the geometric-optical approach, an overlap calculated from the shape of the crowns allows the estimation of the proportion of shadows cast as a function of view direction relative to the hot spot direction. More recently, the GHOST model (Lacaze and Roujean, 2001b) was developed to address the local scale angular structure of the hot spot, which can be judged relevant for patch and regional scales. Although it was specifically designed to simulate the BRDF of boreal forests, it is also suitable to study simpler vegetation canopies like crops and pasture (Lacaze et al., 2002). This study proposes an operational approach, namely DISMA (DIrectional SMA), to deconvolve the angular reflectance into single landcovers reflectances, attempting to solve the inconsistencies of 1D models and linear mixture approaches. The model formalism and the volume scattering formulation are similar to the GHOST model. However, the between-crown gap probability is formulated in terms of the fractional vegetation cover ( $f$ Cover) and a geometric variable $(\eta)$ associated with the shape of plants. A random spatial distribution of plants is assumed to simplify its computation. The invertibility of the model to retrieve LAI was demonstrated using airborne POLDER and HyMap multi-angular measurements corresponding to cropland. The next section describes the model formulation. The inversion algorithm is presented in Section 3. The model is validated in Section 4. Finally, the conclusions and future prospects are presented in Section 5 .

\section{Model formulation}

The scene consists of subcanopies idealized by geometric elements superposed on a flat background. The reflectance of an individual pixel is assumed to consist of an area weighted linear combination of the soil and vegetation contributions. The vegetation reflectance is expressed as the sum of single scattering ( $s s)$ and multiple scattering $(\mathrm{ms})$ reflectances (Hapke, 1981; Lacaze and Roujean, 2001b)

$$
R_{v}=\frac{\omega}{4} \frac{1}{\mu_{s} \mu_{v}} \cdot\left\{P(\xi) I_{s s}+\left[H\left(\mu_{s}\right) H\left(\mu_{v}\right)-1\right] I_{m s}\right\}
$$

where leaf albedo $\omega$ is the sum of the leaf reflectance $(\rho)$ and transmittance $(\tau), \mu_{s, v}=\cos \theta_{s, v}$, $\xi$ is the phase angle (i.e. the angle between the sun and the viewer), and the factors $I_{s s}$ and $I_{m s}$ model the proportion of radiation flux which is single/multiple scattered by foliage elements on the downgoing and outgoing optical pathways as a whole, $H\left(\mu_{s, v}\right)$ is the Chandrasekhar function for multiple scattering (Hapke, 1981) and $P(\xi)$ is a turbid medium phase function (Ross, 1981; Lacaze and Roujean, 2001b). The GHOST model relies on the coupling between a simple hot spot formula (Roujean, 2000) and the $G$ function that describes the canopy geometry. In our model, $G$ controls only the volume component depending on the within-crown element distribution, whereas the external geometric component depending on the crown shape and dimension is evaluated using an average theory of the gap probability. We assume that the geometrical component and the volume component of the radiation fluxes can be decoupled

$$
\begin{aligned}
& I_{s s}=I_{s s, \text { vol }} \cdot I_{s s, \text { geo }} \\
& I_{m s}=I_{m s, \text { vol }} \cdot I_{m s, \text { geo }} .
\end{aligned}
$$

The volume single/multiple scattered component can be described using Beers' Law

$$
\begin{aligned}
I_{s s, \text { vol }} & =\frac{1}{\Delta}\left[1-\exp \left(-\Delta \mathrm{LAI} \Omega_{E} \gamma / g_{c}\right)\right] \\
I_{m s, \text { vol }} & =\frac{1}{\Delta^{\prime}}\left[1-\exp \left(-\Delta^{\prime} \mathrm{LAI} \Omega_{E} \gamma / g_{c}\right)\right]
\end{aligned}
$$

where $\Omega_{\mathrm{E}}$ is the clumping index of the shoots, which quantifies the level of foliage aggregation within the tree crown and is generally dependent on the view angle (Kucharik et al., 
1997); $\Omega_{E}=1$ means random foliage distribution and $\Omega_{E}<1$ means clumped foliage; LAI is the total canopy overstorey leaf area index; $g_{c}$ denotes the $f$ Cover and $\gamma$ is a band-specific factor, which was assumed to be dependent on the leaf transmittance (e.g., Bégué, 1993). Assuming plants with similar foliar density in the scene, $\mathrm{LAI} / g_{c}$ represents a mean value of the LAI of individual plants. This term is more pertinent to describe crown trees transparency than total LAI. $\Delta$ denotes the (bidirectional) normalised extinction coefficient for singly scattered radiance. The model adopts the analytical expression derived by Roujean (2000) to the hot spot effect, i.e. coupling the downgoing and outgoing optical pathways

$$
\begin{aligned}
\Delta & =\sqrt{\frac{G_{s}^{2}}{\mu_{s}^{2}}+\frac{G_{v}^{2}}{\mu_{v}^{2}}-2 \frac{G_{s}}{\mu_{s}} \frac{G_{v}}{\mu_{v}} \cos \xi} \\
\Delta^{\prime} & =\frac{G_{s}}{\mu_{s}}+\frac{G_{v}}{\mu_{v}}
\end{aligned}
$$

where $G$ is the well-known Ross function (Ross, 1981). For the multiple scattered component the hot spot phenomenon is ignored (Qin and Goel, 1995).

The geometric component of the single scattering $I_{s s, \text { geo }}$ is determined by the between-crown light penetration and the visibility of illuminated objects. This component is particularly relevant for discontinuous canopies. The downgoing and outgoing terms of the intercepted flux were formulated in terms of horizontal projection of the crown. Let $P_{0}(\theta)$ denote the (between-crown) monodirectional gap fraction, which corresponds to the fraction of soil seen in the direction $\theta$ (Nilson, 1971). For homogeneous Poisson distribution, the probability of observing the ground under the tree crowns in any given pixel approaches to $P_{0}\left(\theta_{s}\right)=\left(1-g_{c}\right)^{\left(\eta_{s}+1\right)}$ (Jasinski and Eagleson, 1989), where $\eta$ is defined as the ratio of ground projected shadow to plant area. It absorbs all the geometric factors that relate canopy area to shadowing area into only one variable. Its analytical expression for the most common geometrical bodies is provided in Jasinski and Eagleson (1990). The probability of having sunlit ground/ crown $\left(P_{i g, c}\right)$ and viewed ground/crown $\left(P_{v g, c}\right)$ can be expressed as follows:

$$
\begin{gathered}
P_{v g}=P_{0}\left(\theta_{v}\right) \\
P_{i g}=P_{0}\left(\theta_{s}\right) \\
P_{v c}=1-P_{0}\left(\theta_{v}\right) \\
P_{i c}=1-P_{0}\left(\theta_{s}\right) .
\end{gathered}
$$

A functional relationship can also be found between subpixel shaded ground $P_{s g}$ and fractional vegetation cover

$$
P_{s g}=1-g_{c}-\left(1-g_{c}\right)^{\left(\eta_{s}+1\right)} .
$$

This equation is applicable at large sampling scales, when imaging stands resolutions greater than the size of the tree crowns, as confirmed in a preliminary analysis using simulated data (García-Haro et al., 2002). The probability of observing sunlit crown when $P_{i c}$ and $P_{v c}$ are not correlated is simply the product of both probabilities $P_{i c} P_{v c}$, where $P_{i c}$ controls the amount of light intercepted by crowns and $P_{v c}$ controls the contribution of visible crowns to the scene radiance. However, hot spot kernels are necessary to account for the correlation between the two gap probabilities along sun and view directions (Chen and Leblanc, 1997; Qin and Goel, 1995). We assume that the hot spot has a minor influence on the multiple scattered interception at a crown level, i.e. $I_{m s, \text { geo }}=P_{i c} P_{v c}$, but introduce a hot spot kernel $F_{c}$ to modulate the dependence between the optical paths for the single scattered radiation

$$
I_{s s, \text { geo }}=P_{i c} P_{v c}+\left[P_{i c}-P_{i c} P_{v c}\right] F_{c}
$$

where $F_{c}$ is usually obtained from the overlap function between viewing and illuminated shadows as projected on the background ( $\mathrm{Li}$ and Strahler, 1992; Schaff et al., 1994).

Finally, the soil contribution $R_{s}$ is expressed as the product of the background bidirectional reflectance $\gamma_{s}$ and the vegetation transmittance $T$. The vegetation transmittance is the sum of the probability that a solar ray beam will reach the ground without intercepting any crown $T_{\text {geo }}$ plus the probability of intercepting a crown without hitting any foliage element $\left(1-T_{\mathrm{geo}}\right) T_{\mathrm{vol}}$, i.e. $T=T_{\mathrm{geo}}+\left(1-T_{\mathrm{geo}}\right) T_{\mathrm{vol}}$. The following expressions were considered for the transmissions: 


$$
\begin{aligned}
& T_{\text {vol }}=\exp \left(-\Delta \mathrm{LAI} \Omega_{E} \gamma / g_{c}\right) \\
& T_{\text {geo }}=P_{i g} P_{v g}+\left(P_{i g}+P_{i g} P_{v g}\right) F_{G}
\end{aligned}
$$

where $F_{G}$ is a hot spot kernel. It tends to zero as the sun and view directions are far apart $\left(\xi>>0^{\circ}\right)$, i.e. when the viewer sees the sunlit ground through a gap different from that of illumination. The hot spot gradient function also decreases as the view zenith angle approaches a horizontal view perspective $\left(\theta_{v} \rightarrow 90^{\circ}\right)$ and as the leaf density decreases. In this work we have used hot spot kernels $F_{G}$ and $F_{c}$ similar to the hot spot function proposed by White et al. (2001), which match the hot spot region measured in many BOREAS sites (Leblanc et al., 1997).

Our model neglects side scattered diffuse radiation incident on ground surface after multiple scattering with leaves of neighbouring plants, which can be significant over a bright background. The model is thus less accurate at near-infrared wavelengths at which multiple scattering in plant canopies is the strongest within the solar spectrum. Considering also the presence of skylight irradiance, the reflectance can be expressed as a sum of the direct and isotropic diffuse components, where the component of diffuse reflectance is calculated as the average of the measurements in the principal plane. In addition, the model allows us to derive other parameters like the entire BRDF distribution, albedo or absorptance, including the relative contribution of vegetation and soil.

\section{Model inversion}

The inversions are achieved simultaneously for all spectral bands, i.e. by coupling the spectral and directional data available. The variables to be retrieved are LAI and $f$ Cover. Without $a$ priori information on these variables, the inverse problem typically consists in determining the optimal set of variables that minimizes the distance between observations and modelled values. Given BRDF values $r_{i}(i=1, \ldots, N)$, representing the conditions of observation (i.e. wavelengths and view and illumination geometries), the retrievals are performed by comparing observed and modelled $y_{i}(i=1, \ldots, N)$
BRDF's. The comparisons are evaluated for a full set of prescribed canopy realisations ( $f \mathrm{Cov}-$ er, LAI) that cover a range of expected natural conditions. Those pairs for which the canopy model generates inputs comparable with measured data within the limits of accuracy, i.e.

$$
(\boldsymbol{r}-\boldsymbol{y})^{T} \boldsymbol{W}^{-1}(\boldsymbol{r}-\boldsymbol{y}) \leq N
$$

are considered acceptable solutions, where $\boldsymbol{W}$ is the covariance matrix of the measurements, which accounts for both data and model uncertainties. The distribution of solutions defines a domain for $f$ Cover and LAI around the «true» values. Weighted mean values of $f$ Cover and LAI averaged over the set of acceptable solutions are taken as solutions. This procedure not only increases the numerical stability of the inversion but also enables us to derive the uncertainty and correlation of derived parameters. Although secondary model parameters (LAD, clumping, plant dimensions) can be optimised based on prior expected values for each different cover type, in this study they were assumed constant in order to represent a common situation found in global studies (i.e. absence of accurate land cover classification, presence of mixed pixels, etc.). We must note, however, that prior information about the most probable solution values, their uncertainty and their correlation may significantly improve the accuracy of the retrievals, especially for ill posed inversion problems (Combal et al., 2002). In order to reduce the computations, we used an automatic method to select the solution bounds. Firstly, spectral mixture analysis was applied to obtain $f$ Cover from anisotropically corrected reflectance using as input endmembers representing soil and vegetation. Secondly, an empirical relationship was used to derive LAI from estimated $f$ Cover (Lacaze and Roujean, 2001a). The estimated values for LAI and $f$ Cover were finally used to define the bidimensional bounds in the solutions domain.

The model requires the optical properties of the underlying soil as $\gamma_{s}$ an input. We employed the RPV model (Rahman et al., 1993) to extend the BRF measured at a few angles to the entire BRDF distribution. A variable configuration using two different soil BRDFs was used to represent more realistically the influence on reflec- 

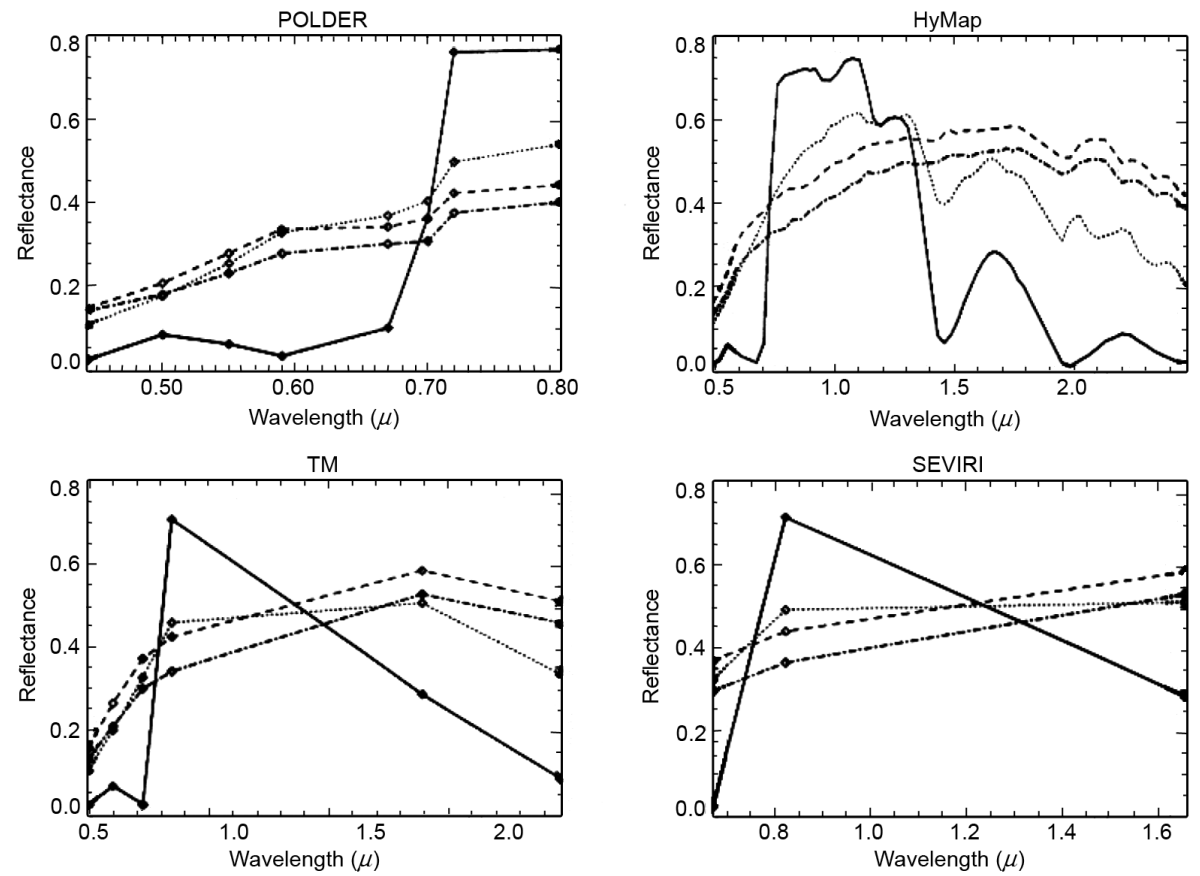

Fig. 1. Spectral inputs introduced to DISMA, which correspond to leaf albedo of green (solid) and dry vegetation (dotted), and reflectance of two different soils.

tance of soil moisture and roughness (see fig. 1). Although leaf reflectance and transmittance are not equal in many plants and vary greatly between species, it was assumed for simplicity that they are similar. We have proposed a method to estimate the leaf albedo $\omega$ from the data themselves. The method relies on the assumption that pixels presenting a negligible contribution of the soil background (i.e. $f$ Cover $=1$ and high LAI values) can be found in the scene. In these pixels eq. (2.1) is inverted in order to retrieve $\omega$ using an iterative method. Results have indicated the adequate convergence of the method, providing reasonable results irrespectively of the data set considered (fig. 1).

\section{Validation}

The model invertibility was evaluated using data acquired during the 1999 Digital Airborne
Spectrometer EXperiment (DAISEX) campaign by the sensors HyMap onboard the DLR Do-228 aircraft and POLDER onboard an ARAT plane. The experiment site selected by ESA for the DAISEX campaigns is a $4 \mathrm{~km}$ by 4 $\mathrm{km}$ area centred at $39^{\circ} 3^{\prime} \mathrm{N}, 2^{\circ} 5^{\prime} \mathrm{W}$, which is located $28 \mathrm{~km}$ from Albacete (Spain), (Camachode Coca et al., 2002). The POLDER instrument allows a measurement of surface reflectance directional effects at nine spectral bands (centred at 443, 500, 550, 590, 670, 700, 720, 800 and $864 \mathrm{~nm}$ wavelength) in the visible and near infrared. The CCD matrix permits collection of bidimensional images in one shot. The along track and cross track FoV is of $\pm 43^{\circ}$ and $\pm 51^{\circ}$ respectively. Four flights were undertaken during 3-5 June 1999 at a typical airborne altitude of $3000 \mathrm{~m}$, with a spatial resolution of $20 \mathrm{~m}$. Each flight recorded around 140 spectral images. They provide around 50 values of angular reflectance for every pixel, irregularly distrib- 
uted in the viewing hemisphere, and different for every pixel. The images were calibrated, geo-coded and corrected for atmospheric effects as it is specified in Leroy et al. (2001). In this work the BRDF was interpolated for the full range of view angles for every pixel. The BRDF was then retrieved considering uniform sites of $3 \times 3$ pixels $\left(60 \times 60 \mathrm{~m}^{2}\right)$. The model was inverted against a set of bidirectional reflectance factors taken along the principal plane (i.e. the region with the strongest anisotropy) and along the orthogonal plane. Figures $2 a, b$ and $3 a, b$ show a few examples of measured BRF's along with the values predicted by DISMA. The results demonstrate the potential of the model to accurately explain the spectral and angular variations of the data. DISMA captures the essential BRDF features such as bowl shape, backscattering increase in reflectance and broad hot spot, as well as the spectral contrast between soil and vegetation. The model works better in the visible regions, when the first order scattering effects predominate but in the NIR region (band 8) the discrepancies are higher. In this region directional effects are less apparent due to the reduction of contrast between canopy components and the prevalence of multiple scattering. Another source of error is attributable to unquantified influences of foliage clumping.

The HyMap instrument (http://www.hyvista.com/) has 128 spectral bands and a high
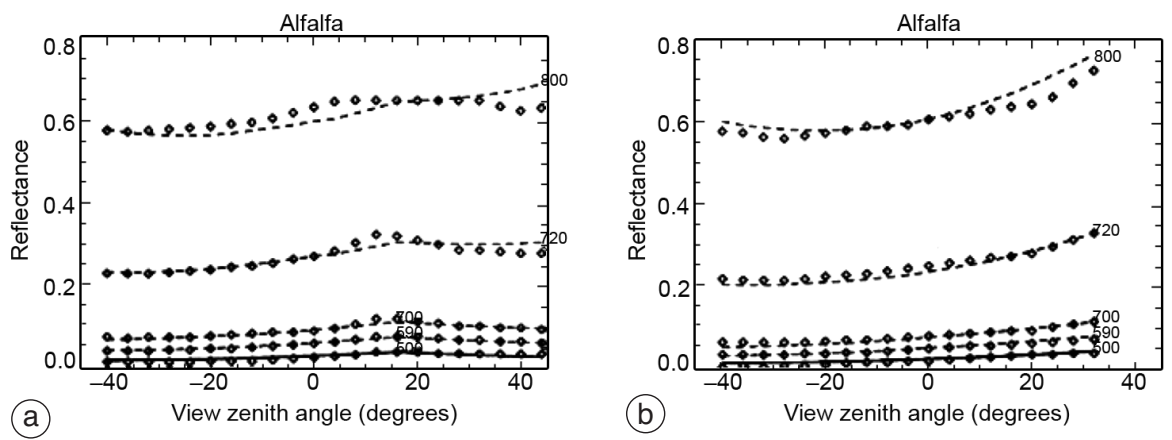

Fig. 2a,b. Comparison of directional signatures in the principal plane measured by POLDER (symbols) and simulated by DISMA (dashed line) at five different bands for alfalfa. a) Noon image $\left(\theta_{V}=17^{\circ}\right)$; b) 14:30 UTC $\left(\theta_{V}=40^{\circ}\right)$.
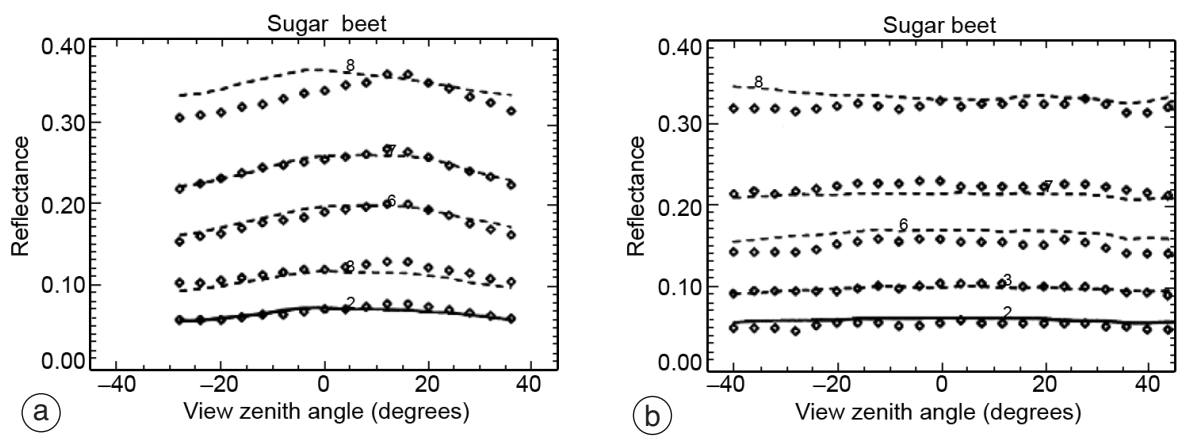

Fig. 3a,b. Idem to fig. 2a,b but considering another angular sampling (the orthogonal plane) and another vegetation type (sugar beet). The numbers $(2,3,6,7$ and 8$)$ refer to the considered POLDER bands. 

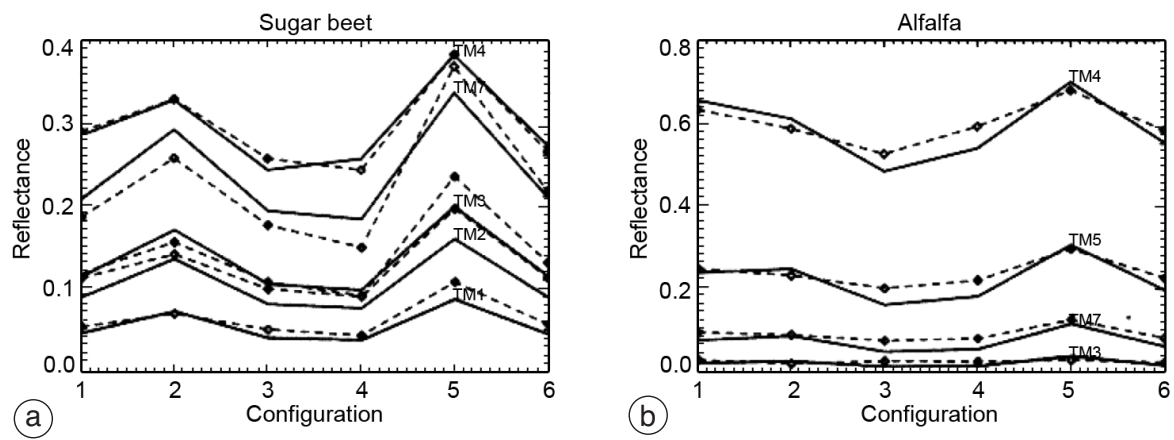

Fig. 4a,b. Measured reflectance (dashed lines + symbols) and simulated (solid lines) at five different spectral channels over sugar beet (a) and alfalfa (b). Configurations 1-3 correspond to the N-S flight and 4-6 to the E-W flight. Within each flight line, consecutive numbers correspond to 8:00, 12:00 and 15:00 UTC, respectively. The figures show several examples of TM-like wavebands derived from real HyMap data.
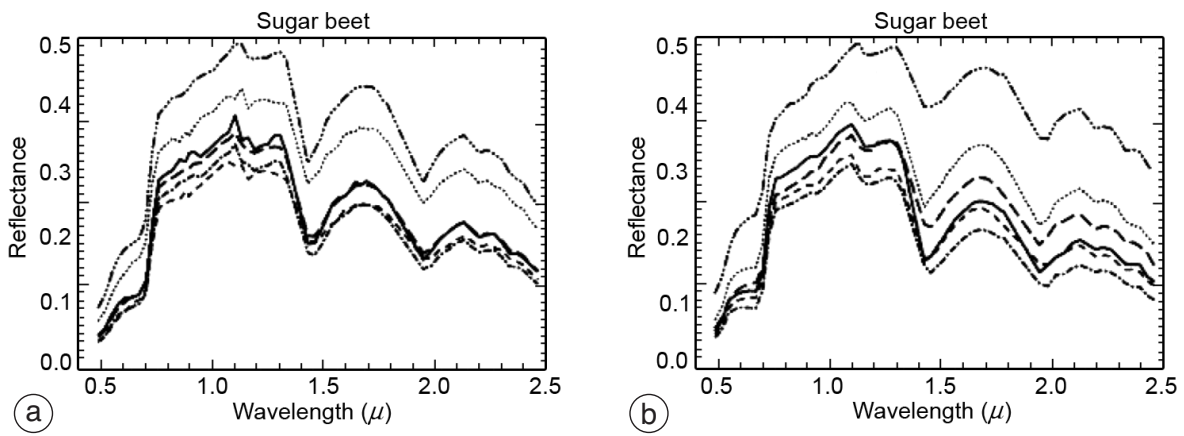

Fig. 5a,b. Angular effects in canopy reflectance of sugar beet associated to the six angular configurations considered in fig. 4a: a) measured HyMap data; b) predicted by the model.

signal-to-noise ratio. To be able to extract the BRDF a complex flight scenario was chosen with data acquisition in two orthogonal flight lines (N-S and E-W) at three different sun geometries: early morning (8:00 UTC), solar noon and late afternoon (15:00 UTC), thus revealing angular reflectance changes also with illumination angle. HyMap data were degraded to POLDER resolution and convolved with the spectral filters of Landsat-5 TM and SEVIRI sensors. Figure $4 \mathrm{a}, \mathrm{b}$ shows two examples of measured BRF's along with the values predicted by DISMA. We can observe that the model addresses the main directional variations found in the data. Figure 5a,b shows another example of the influence of the angular effect on canopy reflectance of discontinuous canopies. We can observe strong differences in the spectral dependence for the different configurations (fig. $5 \mathrm{a}$ ), which are mainly controlled by the directional changes in the proportion of illuminated components, as it is predicted by the proposed model (fig. 5b). These results are indicative of the ability of DISMA not only to extract useful properties of the vegetation but also to reduce the uncertainty of the derived products. Another point of interest in this analysis was to emphasize the usefulness of the diurnal sampling as an angular signature of the surfaces related with the structure of the vegetation cover. 

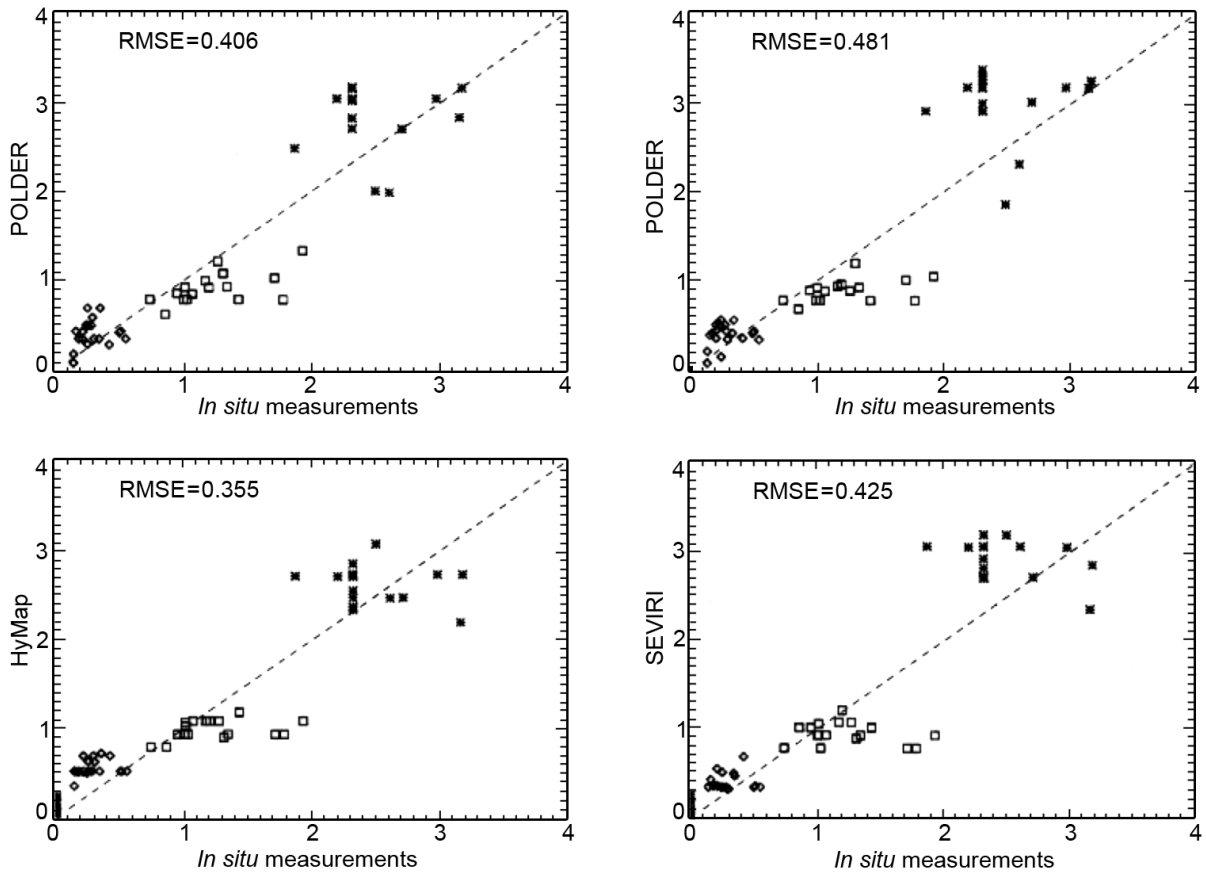

Fig. 6. Relationship between field-measured LAI and airborne-derived LAI using different data sets. The figure at the top corresponds to POLDER data in the principal (left) and orthogonal (right) plane. Different symbols are used to represent corn $(\diamond)$, sugar beet $(\square)$ and alfalfa $(*)$.

Vegetation in situ measurements corresponding to major agricultural units were taken during the campaign. The measured properties included LAI, $f$ Cover, canopy height, biomass and chlorophyll content. The LICOR LAI-2000 instrument was used to measure LAI. Sampling took place at several times along parallel transects inside the fields (García et al., 2001). The comparison between in situ LAI measurements and the retrieved values using different spectroangular datasets is shown in fig. 6. A linear relationship was found in all cases, irrespective of the data set considered. Table I shows the coefficients of the linear fit, revealing a significantly high correlation, with $r^{2}$ values higher than 0.92 in all cases. A chi-square $\left(\chi^{2}\right)$ test was performed assuming a LAI uncertainty of 0.4 . It produced values below the chi-square critical value of 92 in all cases (for 71 degrees of freedom and a probability of 0.05 ), confirming thus the predicted linear relationship. Moreover, re-
Table I. Statistics of the correlation between in situ measurements and retrievals of LAI.

\begin{tabular}{cccccc}
\hline \hline Data set & Offset & Slope & RMSE & $r^{2}$ & $\chi^{2}$ prob. \\
\hline $\begin{array}{c}\text { POLDER } \\
\text { (principal) }\end{array}$ & 0.007 & 1.05 & 0.406 & 0.933 & 0.97 \\
$\begin{array}{c}\text { POLDER } \\
\text { (orthogonal) }\end{array}$ & -0.03 & 1.12 & 0.481 & 0.926 & 0.06 \\
$\begin{array}{c}\text { HyMap } \\
\text { HyMap }\end{array}$ & 0.19 & 0.89 & 0.355 & 0.957 & 0.05 \\
$\begin{array}{c}\text { TM bands) } \\
\text { SEVIRI }\end{array}$ & 0.06 & 1.04 & 0.425 & 0.927 & 0.29 \\
$\begin{array}{c}\text { TM bands) } \\
\text { TM }\end{array}$ & & & & & \\
\hline
\end{tabular}

sults indicate a good correspondence between field measurements and retrievals, since the linear relationship is close to the $1: 1$ line, and the RMSE is relatively low (0.35-0.48). Another 
Table II. Statistics of the cross-comparison of LAI as retrieved using different data sets.

\begin{tabular}{cccccc}
\hline \hline Data set & Offset & Slope RMSE & $r^{2}$ & $\chi^{2}$ prob. \\
\hline $\begin{array}{c}\text { POLDER } \\
\text { principal versus } \\
\text { orthogonal }\end{array}$ & 0.05 & 0.90 & 0.175 & 0.994 & 1 \\
$\begin{array}{c}\text { HyMap } \\
\text { Hersus POLDER }\end{array}$ & -0.18 & 1.15 & 0.308 & 0.970 & 0.999 \\
$\begin{array}{c}\text { HyMap } \\
\text { versus TM }\end{array}$ & -0.10 & 1.14 & 0.197 & 0.994 & 1 \\
$\begin{array}{c}\text { HyMap } \\
\text { versus SEVIRI }\end{array}$ & -0.18 & 1.18 & 0.223 & 0.992 & 1 \\
\hline
\end{tabular}

important result is that DISMA is able to produce reasonable results under sub-optimal conditions either in the angular sampling (i.e. taking the orthogonal plane) or with a small number of spectral channels, e.g., SEVIRI wavebands.

We must also note that even though the retrieved LAI reproduces the between crops LAI variability observed in the field measurements very well, it fails to address the within crop variability (e.g., LAI retrievals tend to saturate within each individual crop). One reason for this is that pixel-by-pixel comparison is strongly hampered by the inaccuracies in the georegistration of field measurements and imagery. Another reason is the averaging process performed to the images. One important aspect is the consistency of the retrievals, i.e. its sensitvity with respect to the set of spectro-angular measurements taken in the inversion. The crosscomparison between results from different data sets showed a high consistency between them, with RMSE values typically lower than 0.30 (see table II). Finally, we must indicate that although in situ measurements included only a limited number of $f$ Cover sampling, the retrieved values were highly coherent with the available field information.

\section{Conclusions and prospects}

This study aimed to develop an operational approach to deconvolve the angular reflectance into single landcovers reflectances, attempting to solve the inconsistencies of 1D models and linear mixture approaches. The model relates the spectral and angular variation with the main optical and structural parameters of discontinuous canopies, like LAI. The inversion revealed the model potential to combine spectral and directional information to increase the likely accuracy of the retrievals. Results also indicate the effectiveness of the algorithm using only SEVIRI channels and information of the diurnal sampling as an angular signature of the surfaces. We preferred to simplify the selection of secondary parameters and the inversion algorithm with specific intent that it not to be canopy dependent. However, for the application of DISMA to complex scenarios as in global studies the stratification of scene is convenient in order to optimise the model inputs to the knowledge of ecosystem characteristics, reducing misidentification and saving computations (García-Haro et al., 2003). One important aspect is the sensitivity of retrieved variables to the information used to parameterise vegetation canopy radiative transfer. Although the inversion algorithm was satisfactory, difficulties still arise. For example the estimation of leaf albedo is known to be impaired by canopy-level variables like LAI. Future research is also needed to test the model on BRDF datasets comprising different vegetation types (shrubland, forest).

\section{Acknowledgements}

This work was supported by the projects DAISEX and LSA SAF. J. García-Haro has currently a research position (Ramon y Cajal) from MCyT, Spain. Special thanks are due to the J. Moreno team for providing us with field measurements.

\section{REFERENCES}

BÉGUÉ, A. (1993): Leaf area index, intercepted photosynthetically active radiation, and spectral vegetation indices: a sensitivity analysis for regular-clumped canopies, Remote Sensing Environ., 45, 45-59.

Camacho-de Coca, F., F.J. García-Haro and J. Meliá (2002): Quantitative analysis of cropland's BRDF anisotropy using airborne POLDER data, in Proceed- 
ings of the 1st International Symposium on Recent Advances in Quantitative Remote Sensing, 16-20 September 2002, Torrent, Spain (Universitat de Valencia, Valencia), 303-308.

ChEn, J.M. and S.G. LEBLANC (1997): A four-scale bidirectional reflectance model based on canopy architecture, IEEE Trans. Geosci. Remote Sensing, 35, 1316-1337.

Combal, B., F. Baret, M. Weiss, A. Trubuil, A. Macé, A. Pragnère, R. MYNENI, Y. KNYAZIHIN and L. WANG (2002): Retrieval of biophysical variables from bidirectional reflectance using prior information to solve illposed inversion problems, Remote Sensing Environ., 84, 1-15.

García, J.C., C. Cuñat, F. Montero, A. Brasa, L. Alonso, M.C. GonzÁlez, J.R. Ruiz, C. Martínez, A. PAlaCIOS and J. Moreno (2001): Vegetation Soil Measurements at Barrax (ESA Publication Division SP-499, ESTEC, The Netherlands), 79-87.

García-Haro, F.J., F. Camacho-de Coca and J. Meliá (2002): Retrieval of biophysical parameters using directional spectral mixture analysis, in Proceedings of the 1st International Symposium on Recent Advances in Quantitative Remote Sensing, 16-20 September 2002, Torrent, Spain (Universitat de Valencia, Valencia), 963-970.

García-Haro, F.J, S. Sommer and T. Kemper (2003): Variable Multiple Endmember Spectral Mixture Analysis (VMESMA), Int. J. Remote Sensing (submitted).

HAPKE, B. (1981): Bidirectional reflectance spectroscopy, 1. Theory, J. Geophys. Res., 86, 3039-3054.

JASINSKI, M.F. and P.S. EAGLESON (1989): The structure of red-infrared scattergrams of semivegetated landscapes, IEEE Trans. Geosci. Remote Sensing, 27, 441-451.

JASINSKI, M.F. and P.S. EAGLESON (1990): Estimation of subpixel vegetation cover using red-infrared scattergrams, IEEE Trans. Geosci. Remote Sensing, 28, 253-267.

KuchariK, C.J., J.M. NoRman, L.M. Murdock and S.T. GOwER (1997): Characterizing canopy nonrandomness with a Multiband Vegetation Imager (MVI), J. Geophys. Res., 102 (D24), 29455-29473.

LACAZE, R and J.L. RouJEAN (2001a): Retrieval of biophysical parameters over land based on POLDER directional and hot spot measurements, in Proceedings of the 8th Symposium on Physical Measurements and Signatures in Remote Sensing, Aussois, 487-492.

LACAZE, R. and J.L. RoujeAn (2001b): G-function and Hot SpoT (GHOST) reflectance model. Application to multi-scale airborne POLDER measurements, Remote Sensing Environ., 76, 67-80.

Lacaze, R., J.M. Chen, J.L. Roujean and S.G. LeblanC (2002): Retrieval of clumping index using the hot spot signatures measured by POLDER instrument, Remote Sensing Environ., 79, 84-95.

Leblanc, S.G., P. Bicheron, J.M. Chen, M. Leroy and J. CIHLAR (1997): Investigation of radiative transfer in boreal forests with an improved 4-scale model and airborne POLDER data, IEEE Trans. Geosci. Remote Sensing, 27, 1396-1414.

Leroy, M., O. Hatecoeur, F. Ponchaut, L. Alonso-ChorDA and J. MORENO (2001): The Airborne POLDER Data in the DAISEX'99 Campaign (ESA Publication Division SP-499, ESTEC, The Netherlands), 13-22.

LI, X. and R.A. STRAHLER (1992): Geometrical-optical modeling of the discrete-crown vegetation canopy: effect of crown shape and mutual shadowing, IEEE Trans. Geosci. Remote Sensing, GE-30, 276-292.

NiLson, T. (1971): A theoretical analysis of the frequency of gaps in plant stands, Agric. Meteorol., 8, 25-38.

QIN, W. and S.A.W. GERSTL (2000): 3D scene modeling of semidesert vegetation cover and its radiation regime, Remote Sensing Environ., 71, 197-206.

QIN, W. and N.S. GoEL (1995): An evaluation hotspot models for vegetation canopies, Remote Sensing Rev., 13, 121-159.

Rahman, H., M.M. Verstraete and B. Pinty (1993): Coupled Surface-Atmosphere Reflectance (CSAR) model, Part 1. Model description and inversion on synthetic Data, J. Geophys. Res., 98, 20,779-20,789.

Ross, J.K. (1981): The Radiation Regime and Architecture of Plants Stands (Dr. W. Junk Publishers, Norwell, MA), pp. 391.

Roujean, J.L. (2000): A parametric hot spot model for optical remote sensing application, Remote Sensing Environ., 71, 197-206.

Roujean, J.L., M. Leroy, P.Y. Deschamps and A. Podaire (1992): Evidence of surface reflectance bidirectional effects form a NOAA/AVHRR multitemporal data set, Int. J. Remote Sensing, 13, 685-698.

SCHAFF, C.B., X. Li and R.A. StRAHLER (1994): Topographic effects on bidirectional and hemispherical reflectances calculated with a geometrical-optical canopy model, IEEE Trans. Geosci. Remote Sensing, GE-32, 1186-1193.

VAN-LEEUWEN, W.J.D. and J.L. RouJEAN (2002): Land surface albedo from the synergistic use of polar (EPS) and geo-stationary (MSG) observing systems. An assessment of physical uncertainties, Remote Sensing Environ., 81, 273-289.

White, H.P., J.R. MiLler and J.M. CHEN (2001): Four scale linear model for anisotropic reflectance (FLAIR) for plant canopies, Part I. Model description and partial validation, IEEE Trans. Geosci. Remote Sensing, 39 (5), 1072-1083. 